POETRY

\title{
8:05am
}

\section{Patrick Kilmartin}

She's been up for an hour already,

while I toss away my sleep-in.

Through the walls,

the breakfast rattle of a nine-to-sixer ...

Pre-commute panic: the interest rate of the real working world.

She's got it tough,

my bank-owned girlfriend.

The sound of a key in a lock and a quietly closed door is our only interaction.

A cold coffee ring in a steamy bathroom, her only traces.

Fossils of us for me to find when finally, I rise to the mirror to take a look at myself. 